\title{
Hysteresis Compensation of 3D Printed Sensors by a Power Law Model with Reduced Parameters
}

\author{
Dimitrios Kosmas, Martijn Schouten, Gijs Krijnen \\ Robotics And Mechatronics group, University of Twente, Enschede, The Netherlands \\ Email: gijs.krijnen@utwente.nl
}

\begin{abstract}
We propose a modified Power Law Model [1] for hysteresis compensation. A simplification of the original model, resulting in a lower number of parameters to be estimated, is introduced. It has no nonlinear resistor in the output stage and the nonlinear resistance function in the input section(s) is given by a sinh function resulting in $3 N+2$ parameters for a model with $N$ input stages. A cantilever beam with two symmetric piezoresistive sensors was 3D printed and shown to exhibit hysteretic behavior. The sensor's differential measurements have been used to obtain training and validation data. We present promising fitting results obtained with a single cell model and 5 parameters only. Finally, the inverse model (compensator) is derived and applied to the experimental data in order to strongly reduce the hysteretic nonlinearity.
\end{abstract}

Keywords - 3D-Printing, Hysteresis, Creep, Compensation, Flexible, Soft, Tactile sensor, Power Law, Non-linear

\section{INTRODUCTION}

Rapid growth of Additive Manufacturing (AM) with the possibilities of multi-material printing, along with the recent developments in Soft Robotics has led to the investigation of embedded sensing in soft structures [2]. Fabrication of such sensors has already shown promising results [3], but at the expense of high nonlinearities such as viscoelastic hysteresis [4], [5], hysteretic piezo-resistive response [6] as well as ratedepended phenomena like stress relaxation and creep [5]. For these types of sensors to be incorporated into real-world applications, e.g. for mechatronic purposes, a model that can satisfactorily describe such behavior is needed.

Modeling of hysteretic and rate-dependent phenomena has been the object of an extensive amount of literature since 1935 when F. Preisach introduced his approach of modeling hysteresis in ferromagnetic materials as a sum of infinite rectangular shaped hysteresis operators [7]. Chua \& Stromsoe introduced a Lumped Circuit model of hysteresis consisting of non-linear inductors and non-linear resistors [8]. In 1994 Parodi, Storace \& Cincotti proposed a piece-wise linear (PWL) ladder circuit model [9] in which the only non-linear elements are the resistive ones, in that way eliminating the extra complexity added by the nonlinear inductors. Biggio \& Storace [1] proposed an extended model based on [9], which enables to capture the logarithmic time dependence of the creep relaxation dynamics.

This work was developed within the Wearable Robotics programme, funded by the Dutch Research Council (NWO).

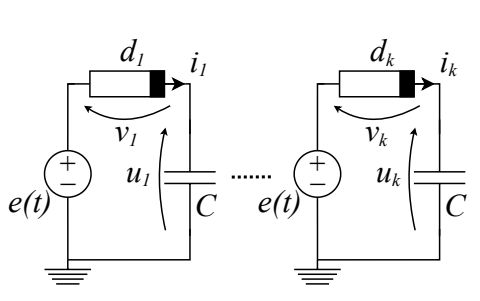

sub-circuit (a)

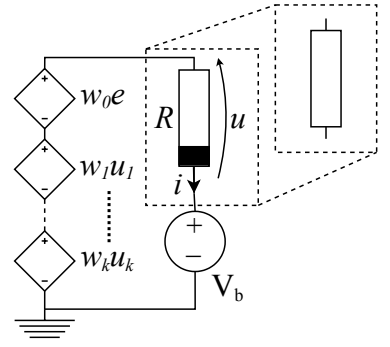

sub-circuit (b)
Fig. 1. Power Law model with the proposed circuit modification shown within the dotted area (top right). Adapted from Fig. 1 of reference [1]

In the current work, we attempt to further reduce the complexity of the model of Biggio \& Storace, by choosing an alternative way to represent the characteristic of the nonlinear resistive elements of the circuit and by changing the fitting procedure. These modifications to the model will first be discussed. Subsequently the model is fitted to measurements obtained on a 3D printed sensor and the inverse of the model is applied to another set of measurement data demonstrating that the model indeed can be used to significantly reduce the hysteresis of a 3D printed sensor.

\section{A. Modification}

\section{MODEL}

The main background of this model is thoroughly explained in [1] and based on the previous work of [9], [10], [11], [12] (and the references therein). Briefly, the Power Law model attempts to model the hysteretic and creep nonlinearities via an electronic circuit model where the hysteretic memory is modelled by a parallel architecture of $1 . . N$ sub-circuits (a), fig 1 . These sub-circuits contain a non-linear resistor $d_{k}$ and a capacitor in series with a voltage source with a voltage equal to the input signal $e(t)$. The sub-circuit (b) contains a set of Voltage Controlled Voltage Sources (VCVSs) of which the voltage depends on either a weighted, by $w_{k}$, version of the voltage $u_{k}(t)$ over the capacitors or the input signal $e(t)$ in the first circuit, and a bias voltage $V_{\mathrm{b}}$. The output signal of the model is the current $i(t)$ going through the non-linear resistor which is in series with the VCVSs. The weights of the VCVS's are estimated through an identification procedure using measurement data. The other elements in the circuit have fixed parameters.

In this work we show that a modified version of this model can be used to reduce the hysteresis and improve the linearity for a sinusoidal input signal using only a single instance of 
the sub-circuit a. This modified version of the model discards the second non-linear element by fixing it to a value of $R=1$ (panel (b)). Also it assumes that the non-linear resistor (panel (a) can be expressed as a weighted hyperbolic sine function

$$
i_{k}=f\left(u_{k}\right)=I_{\mathrm{S}}\left(e^{\frac{u_{k}}{V_{\mathrm{T}}}}-e^{\frac{-u_{k}}{V_{\mathrm{T}}}}\right), \quad k=(1,2, \ldots, N)
$$

Finally we optimise not only the weights $w_{k}$ of the VCCSs, but also the shape of the non-linear resistor by optimizing the parameters $V_{\mathrm{T}}$ and $I_{\mathrm{S}}$. This optimisation is done using a non-linear optimization procedure that finds the minimum of a constrained nonlinear multivariable function [13].

The model can be represented as a non-linear state-space equation

$$
\begin{aligned}
& \dot{u}_{k}=\frac{1}{C} f\left(v_{k}\right)=\frac{1}{C} f\left(e-u_{k}\right) \\
& i R=w_{0} e+\sum_{k=1}^{N} w_{k} u_{k}-V_{b}
\end{aligned}
$$

Hence, the inverse (compensator) output can be represented as

$$
\begin{aligned}
\dot{u}_{k} & =\frac{1}{C} f\left(\frac{i R}{w_{0}}-\sum_{j=1}^{N} \frac{w_{j}}{w_{0}} u_{j}+\frac{V_{\mathrm{b}}}{w_{0}}-u_{k}\right) \\
e & =\frac{i R}{w_{0}}-\sum_{j=1}^{N} \frac{w_{j}}{w_{0}} u_{j}+\frac{V_{\mathrm{b}}}{w_{0}}
\end{aligned}
$$

\section{B. Identification}

In order to estimate the remaining weights $\left(w_{0}, w_{1}, V_{\mathrm{b}}, I_{\mathrm{S}}\right.$, and $\left.V_{\mathrm{T}}\right)$, a set of measurement data is obtained for $\hat{e}(t)$ and $\hat{i}(t)$ (see section III). The number of cells $N$ is to be provided. A cost function is defined as the summed squared error

$$
S S E=\sum_{t=0}^{t_{\text {end }}}(\hat{i}(t)-i(\hat{e}(t)))^{2}
$$

where $\hat{i}(t)$ is the measured, and $i(\hat{e}(t))$ the output modelled for the input $\hat{e}(t)$.

For the minimization, MATLAB's fmincon is used in order to estimate the weights that minimise the cost function defined in 4. An interior-point based algorithm [13] is employed, limited such that the sum of all of the weights is smaller than $10^{2}$.

\section{Verification}

As a measure for the amount of hysteresis of a sensor, the average area encircled in a plot of the normalised input versus the normalised input, will be used. To calculate this area the following equation is used.

$$
\hat{A}=\frac{1}{n} \int_{\hat{x}_{t=0}}^{\hat{x}_{t=n \cdot T}} \hat{y} \cdot d \hat{x}
$$

with:

$$
\begin{aligned}
& \hat{x}=\frac{x-\min (x)}{\max (x)-\min (x)} \\
& \hat{y}=\frac{y-\min (y)}{\max (y)-\min (y)}
\end{aligned}
$$

Where $x$ is the input displacement of the sensor, $y$ is the output signal of the sensor, $T$ is the period of the excitation signal

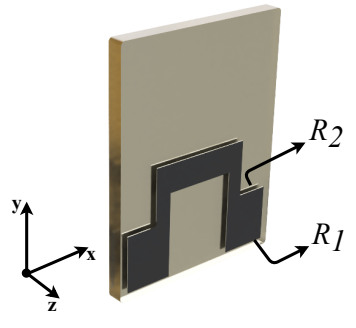

Fig. 2. 3-D CAD render of the differential sensor exposing top $\&$ bottom resistive traces $(\mathrm{co}-$ ordinate frame representing the printing orientation).

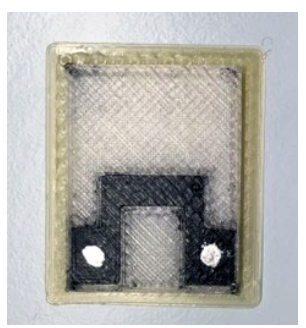

Fig. 3. Fabricated sensor on top of a PVA layer. and $n$ is an integer representing the number of periods in the test signal.

\section{MEASUREMENTS}

\section{A. Sensor}

The sensor fabrication was largely based on the design of the previous work of [3], where it was demonstrated that differential measurements can improve the linearity of the sensor. Here we follow the same concept by implementing two symmetric piezo-resistive elements on both the top and bottom faces of the sensor (fig. 2). The piezo resistors are based on a carbon black filled thermoplastic polyurtherane (TPU) by Ninjatek called EEL [14], as shown in figure 3.

In contrast to the previous sensor, the sensor used in the current work was printed on a raft of PVA. This was done because the first layer often has different properties than the succeeding layers, which causes asymmetry in the sensor.

The measured resistances of the piezo-resistive traces, after allowing a settling period, were found to be $22.4 \mathrm{k} \Omega$ (top) and $15.7 \mathrm{k} \Omega$ (bottom).

\section{B. Set-up}

The sensor is mechanically loaded using a linear actuator (SMAC LCA25-050-15F) running a position control loop generating a sine with a frequency of $0.5 \mathrm{~Hz}$ and an amplitude of $3 \mathrm{~mm}$. To read out the sensor, it is placed in a half-bridge

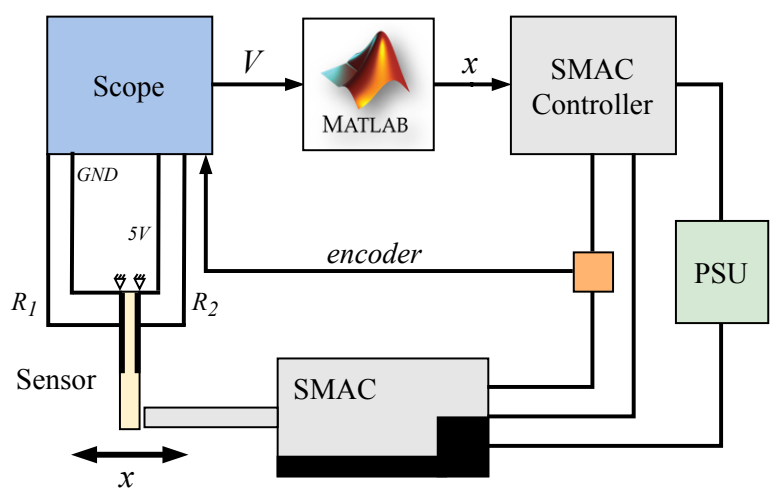

Fig. 4. Overview of the experiment set-up (orange box represents the splitter cable for the extraction of encoder's channels A \& B signals). 


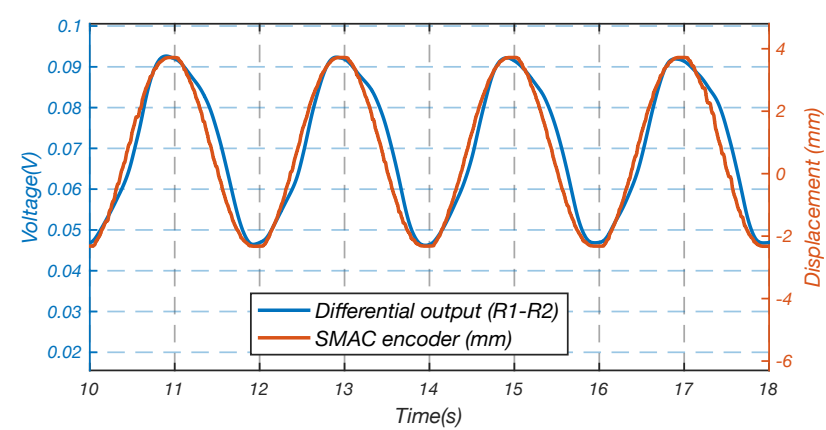

Fig. 5. Differential measurement(left y-axis) lags the commanded commanded position (right $\mathrm{y}$-axis).

configuration using a $10 \mathrm{k} \Omega$ resistor and a $2 \mathrm{~V}$ DC voltage source. The half-bridge output voltage is measured using a Picoscope 5443B running at a sample frequency of $2 \mathrm{MHz}$. In order to be able to guarantee a perfect time synchronization of the half bridge measurement with the position readout, the encoder signal from the SMAC actuator controller is extracted and read by the PicoScope.

At the sensor channels, a down-sampling ratio of 5 along with a digital zero-phase low-pass $2^{\text {nd }}$ order Butterworth filter is applied with a cut-off frequency of $f_{c}=20 \mathrm{~Hz}$. Due to an observed high-frequency interference of the SMAC controller, a hardware RC low-pass filter is added with $R=10 \mathrm{k} \Omega$ and $C=15 \mathrm{nF}$. An overview of the setup is presented in fig 4 .

\section{RESUlTS}

Two data sets of $\hat{e}(t)$ (SMAC position) and $\hat{i}(t)$ (half bridge output voltage) were obtained by two distinct experiments of 5 min duration each. Cross-validation is used, with one set of data for fitting and one for validation respectively. The differential measurement versus the input position, see figure 5, is forming the hysteresis loop. Using the measured time series $\hat{e}(t)$ and $i(t)$ the model is nonlinearly fitted with the first set. For the computation, sub-circuit (a) is comprised with a single cell $(N=1)$. The capacitance is fixed at $C=1 \mathrm{~F}$. The initial conditions are given as: $I_{\mathrm{S}}=0.1, V_{\mathrm{T}}=0.2, w_{0}=1, w_{1}=0.4$ and $V_{\mathrm{b}}=-0.6$. Hence, a total of five parameters are to be optimized through the optimization process. The model fit is shown in fig 6 .

The inverse model, given the optimized parameters, is applied to the second set of data and the output is plotted against the experiment data in fig 7 . The SINAD and the normalised hysteresis when compared to the SMAC encoder signal as defined in equation 5 are calculated for the most important signals (Table I).

\section{DISCUSSION AND CONCLUSIONS}

The modified model shows a good fit against the experimental data, correctly representing the hysteretic nonlinearity. MATLAB's fmincon proves that a good fit is possible. However a more efficient, in terms of computation time, approach is likely required before the model can be adapted in an online estimation.

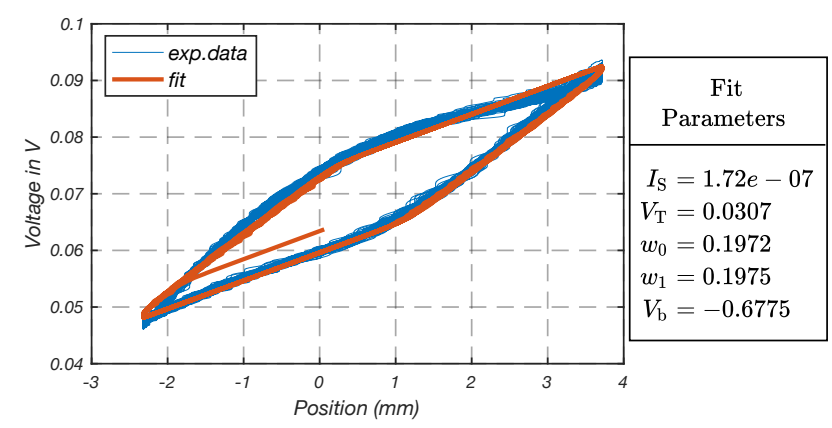

Fig. 6. Experimental data and Modified PL model fit.
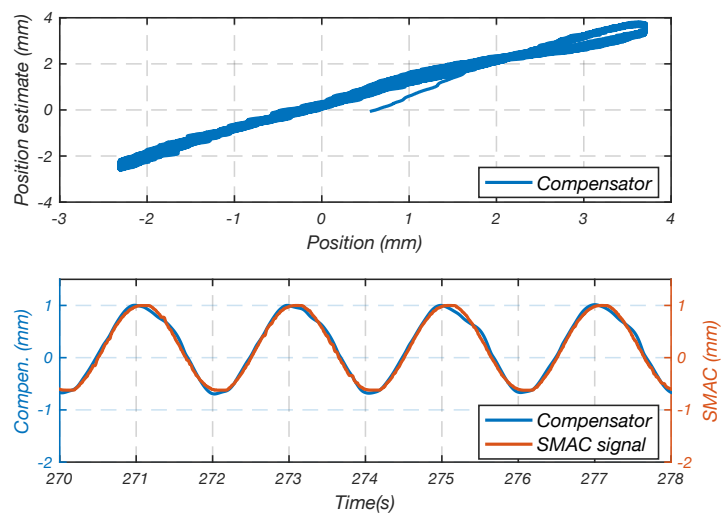

Fig. 7. Inverse model output versus measured position and response of the compensator.

Furthermore, the inverse model is applied and is shown to provide a good estimation of the original position (fig. 7-top). Subsequently, the response of the compensator is shown to reduce the hysteretic non-linearity (fig. 7-bottom-) as compared to fig. 5. As far as the linearity is concerned, only a slight improvement was observed. (table I).

In this experiment, an empirical model is constructed for a system that consists of both a cantilever beam fabricated out of a viscoelastic material as well as a piezo-resistive sensing element. In order to be able to model these elements separately the force applied on the cantilever should be measured as well.

Further research is required in order to validate the simplified model's performance. The presented results hold for a specific frequency and amplitude. For different cases the need to employ a larger number of $N$ cells might be necessary. The ability to represent inner loops and the model's performance for excitation at different frequencies and amplitudes is still to be investigated. Preliminary results are promising, albeit at the expense of requiring multiple cells instead of one.

TABLE I. SINAD

\begin{tabular}{lcc}
\hline Signal & SINAD $(\mathrm{dB})$ & $\hat{A}(\%)$ \\
\hline SMAC encoder signal & 32.2 & 0 \\
$R_{1}$ & 4.9 & -10 \\
$R_{2}$ & 5.7 & 15 \\
$R_{1}-R_{2}$ & 18.4 & -18 \\
Fit & 23.6 & -20 \\
Compensated & 20.6 & 0.8 \\
\hline
\end{tabular}




\section{REFERENCES}

[1] M. Biggio, A. Oliveri, F. Stellino, M. Parodi, and M. Storace, "A circuit model of hysteresis and creep," IEEE Transactions on Circuits and Systems II: Express Briefs, vol. 62, no. 5, pp. 501-505, 2015.

[2] A. Dijkshoorn, P. Werkman, M. Welleweerd, G. Wolterink, B. Eijking, J. Delamare, R. Sanders, and G. J. Krijnen, "Embedded sensing: Integrating sensors in 3-D printed structures," Journal of Sensors and Sensor Systems, vol. 7, no. 1, pp. 169-181, 2018.

[3] M. Schouten, B. Prakken, R. Sanders, and G. Krijnen, "Linearisation of a $3 \mathrm{~d}$ printed flexible tactile sensor based on piezoresistive sensing," in 2019 IEEE SENSORS, Oct 2019, pp. 1-4.

[4] H. Banks, "A brief review of some approaches to hysteresis in viscoelastic polymers," Nonlinear Analysis: Theory, Methods \& Applications, vol. 69 , no. 3, pp. 807-815, 2008.

[5] H. Qi and M. Boyce, "Stress-strain behavior of thermoplastic polyurethanes," Mechanics of Materials, vol. 37, no. 8, pp. 817-839, 2005.

[6] J. Shintake, E. Piskarev, S. H. Jeong, and D. Floreano, "Ultrastretchable strain sensors using carbon black-filled elastomer composites and comparison of capacitive versus resistive sensors," Advanced Materials Technologies, vol. 3, no. 3, p. 1700284, 2017.

[7] F. Preisach, "Uber die magnetische nachwirkung," Zeitschrift fur Physik, vol. 94, no. 5-6, pp. 277-302, 1935.

[8] L. Chua and K. Stromsmoe, "Lumped-circuit models for nonlinear inductors exhibiting hysteresis loops," IEEE Transactions on Circuit Theory, vol. 17, no. 4, pp. 564-574, 1970.

[9] M. Parodi, M. Storace, and S. Cincotti, "A pwl ladder circuit which exhibits hysteresis," International Journal of Circuit Theory and Applications, vol. 22, no. 6, pp. 513-526, 1994.

[10] S. Bobbio and G. Marrucci, "A possible alternative to preisach's model of static hysteresis," Il Nuovo Cimento D, vol. 15, no. 5, pp. 723-733, 1993.

[11] M. Storace and M. Parodi, "On the representation of static hysteresis curves by a pwl ladder circuit," International Journal of Circuit Theory and Applications, vol. 26, no. 2, pp. 167-177, 1998.

[12] A. Oliveri, M. Lodi, F. Stellino, and M. Storace, "Modeling and compensation of hysteresis and creep: The hystool toolbox," 2018 IEEE International Symposium on Circuits and Systems (ISCAS), 2018.

[13] "Matlab optimization toolbox," Version 8.4 (R2019b), mathWorks, Natick, MA, USA.

[14] Fenner Drives, "Eel 3d printing filament," Report, 2018. [Online]. Available: http://ninjatek.com/wp-content/uploads/2018/12/Eel-TDS.pdf 